\title{
Influence of Vibration and Heat Treatment on Residual Stress of a Machined $12 \% \mathrm{Cr}$-Steel
}

\author{
Ru Lin Peng ${ }^{1, a^{*}}$, Johan Moverare ${ }^{1,2, b}$, Pajazit Avdovic ${ }^{2, c}$, Annethe Billenius ${ }^{1, d}$ \\ and Zhe Chen ${ }^{1, \mathrm{e}}$
}

${ }^{1}$ Department of Management and Engineering, Linköping University, Linköping, Sweden

${ }^{2}$ Siemens Industrial Turbomachinery, S-612 83 Finspong, Sweden

aru.peng@liu.se, bjohan.moverare@liu.se, 'pajazit.avdovic@siemens.com, dannethe.billenius@liu.se, e'zhe.chen@liu.se

Key words: Vibratory stress relief, Thermal stress relief, Machining, Residual stress, $12 \%$ Cr-steel

\begin{abstract}
In this paper we investigated the influence of vibratory stress relieving technique, which is widely used for stress relaxation of weld and casting components/structure, on machining residual stresses in a ring-component of $12 \% \mathrm{Cr}$-steel. It was shown that the employed vibratory treatment, without significantly altering the microstructure, turned the surface layer from tension into compression but retained the compressive residual stresses in the subsurface. In comparison, a stress relieving heat treatment, included as a reference in the study, removed completely the surface tensile residual stresses and reduced the subsurface compressive residual stresses to a low level. Significant microstructural changes in the form of recrystallization also occurred in a thin surface layer of the machining affected zone after the heat treatment.
\end{abstract}

\section{Introduction}

Machining of metallic components induce both local heating and plastic deformation which result in the generation of residual stresses in a surface layer of the machined components. For many applications residual stresses may not be desirable. For example, they can affect directly the dimensional stability in further manufacturing processes or during service. For the mechanical behavior, tensile residual stresses are often considered to be detrimental as they may promote the initiation of fatigue crack and accelerate crack propagation. Surface tensile residual stresses can also increase the tendency for stress corrosion cracking. Therefore, it is important to reduce or eliminate the undesired residual stresses from machining operations.

The thermal stress relaxation (TSR) method is commonly used to reduce residual stresses from different manufacturing processes. By keeping the component at an elevated temperature for a certain time period, often a few hours, residual stresses are reduced to a significantly low level through movement of dislocations [1]. In comparison with TSR, the vibratory stress relief (VSR) technique, which has a much shorter history, has been used mostly for castings, welds and other components/structures containing thermal residual stresses [2,3]. During the VSR treatment, the component/structure is fixed on a rubber pad connecting to a vibrator. The vibratory frequency is increased to reach a resonant frequency. Relaxation of the residual stresses is attributed to a large peak stress, the sum of stress amplitude from vibration and local residual stress, that induces local plasticity [4-6]. The main advantages of VSR over TSR include higher process efficiency, lower energy consumption, no surface oxidation, feasibility for large components, etc. For example, the VSR treatment of cast cylinder wallboards in carding machine takes 20 minutes with $95 \%$ less energy consumption in comparison with the TSR treatment which requires 20 hours [7].

Different from castings and welds, residual stresses in machined components are distinguished by high peak stresses that change sharply over a small depth near the surface region. While it is not certain how such a localized stress field would be affected by a vibration load, very few reports dedicated to VSR treatments of machined component could be found. In this paper, we present our investigations on residual stresses and microstructure of a VSR treated ring of $12 \% \mathrm{Cr}$-steel 
manufactured by turning. The X-ray diffraction technique was used to measure residual stresses and scanning electron microscopy was carried out to study related microstructural changes induced by the VSR process. A comparison to the influence of a standard TSR treatment was also made. The results show that in contrast to the TSR method, which altered significantly the microstructure in a surface layer, the VSR treatment effectively removed the surface tensile residual stresses without introducing a strong influence on the microstructure.

\section{Experimental Details}

The nominal chemical composition of the $\mathrm{X} 22 \mathrm{CrMoV} 12-1$ steel is: $0.22 \% \mathrm{C}, 0.3 \% \mathrm{Si}, 0.65 \% \mathrm{Mn}$, $11.5 \% \mathrm{Cr}, 1.0 \% \mathrm{Mo}, 0.6 \% \mathrm{Ni}, 0.3 \% \mathrm{~V}$ and rest in $\mathrm{Fe}$. The material was heat treated by austenizing at $1120{ }^{\circ} \mathrm{C}$, quenching in oil to a martensitic structure and finally tempering at $700{ }^{\circ} \mathrm{C}$ for 2 hours. The tempered martensitic microstructure consists of lath ferrite and fine carbides distributed often at the lath boundaries and prior austenite boundaries (Fig. 1). Part of the shear deformation involved in the martensitic transformation remains after the tempering treatment and is visible as subgrains in some laths (Fig. 1(b)) but in some other laths little strain contrast (Fig. 1(c)) is observed. The nominal yield strength of the steel in the tempered condition is over $700 \mathrm{MPa}$ and the nominal tensile strength is $900-1050 \mathrm{MPa}$.
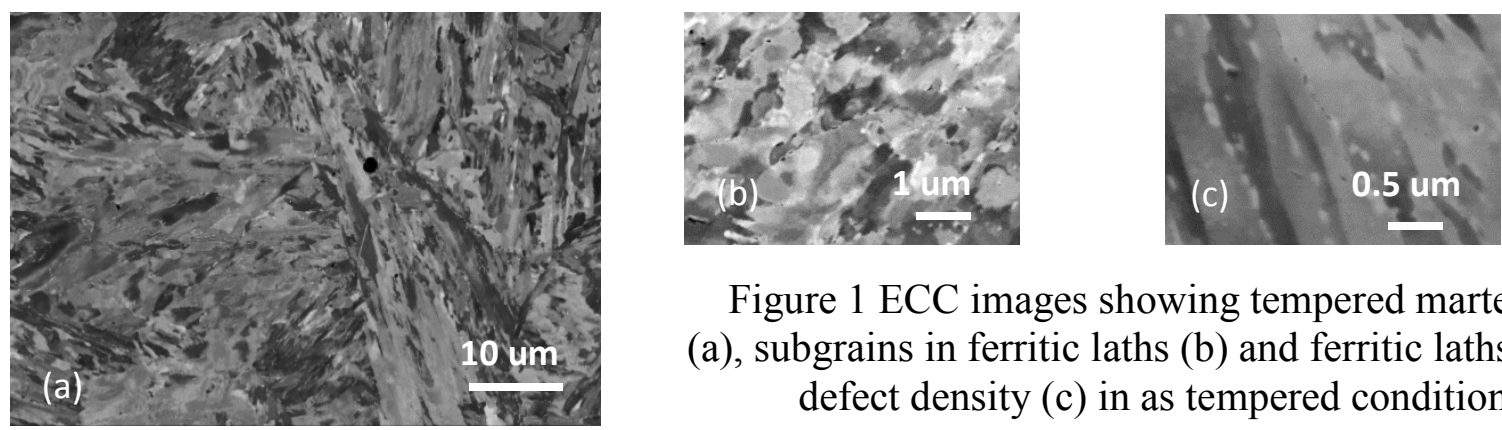

Figure 1 ECC images showing tempered martensite (a), subgrains in ferritic laths (b) and ferritic laths of low defect density (c) in as tempered condition.

Ring components of $280 \mathrm{~mm}$ in inner diameter, $300 \mathrm{~mm}$ in outer diameter and $10 \mathrm{~mm}$ in thickness were manufactured by wet turning in a Max Muller machine. One of the two flat surfaces of the ring to be examined was prepared using cutting depth $\mathrm{a}_{\mathrm{p}}=1 \mathrm{~mm}$, feeding $\mathrm{f}=0.4 \mathrm{~mm} / \mathrm{rev}$ and speed determined by $\mathrm{S}=150 \mathrm{rev} / \mathrm{min}$. The tool holder is PCLNL3232P16 and insert CNMG160608QM4225. A vibratory stress relief treatment was carried out on one of the rings by fixing it on rubber pads and vibrating through a rotary vibrator at $85 \mathrm{~Hz}$ for 30 minutes. Another ring was subjected to a standard stress relief annealing, namely $660{ }^{\circ} \mathrm{C}$ and 5 hours.

Slices were taken from the rings for experimental characterization. For microstructural studies, a cross-section perpendicular to the cutting direction (CD) was mechanically polished following a procedure for preparing surfaces for EBSD examination. The examination was performed by electron channeling contrast (ECC) imaging and electron backscatter diffraction (EBSD) mapping in a Hitachi SU-70 FEG-SEM (field emission gun - scanning electron microscope).

The XRD analyses were made on the prepared flat surface of the rings. Residual stresses along the cutting (the tangent direction of the ring) and the feed (the radial direction of the ring) were evaluated using a Seifert 4-axis diffractometer with $\mathrm{Cr}-\mathrm{K}_{\alpha}$ radiation and a $\Phi 2 \mathrm{~mm}$ collimator. The standard $\sin ^{2} \psi$ method [8] was used with 7 tilting angles spreading between $\psi=+55^{\circ}$ and $\psi=-55^{\circ}$. Diffraction peaks from the $\{211\}$ planes of the matrix BCC phase were obtained and fitted with a Pseudo-Voigt function to determine the peak positions, $2 \theta$, and peak width, FWHM, of the $\mathrm{K}_{\alpha}$ doublets. The diffraction elastic constant for calculation of residual stresses is $5.81 \times 10^{-6} \mathrm{MPa}^{-1}$. To obtain the depth profile, layers of material were removed stepwise by electrolytic polishing. The residual stress profiles presented below are the as measured values without any correction for possible stress relaxation. 


\section{Results and discussions}

\subsection{Residual Stresses}

Results from the XRD measurements are given in Fig. 2. The machining operation has induced tensile stresses in a thin surface layer and compressive stresses in a much thicker subsurface layer (Fig. 2(a)). The stress component in the feed direction (FD) decreases rapidly from $460 \mathrm{MPa}$ to zero within a depth of $15 \mu \mathrm{m}$. The subsurface compressive zone extends to a depth of about $200 \mu \mathrm{m}$ with a rather flat subsurface maximum of about $-340 \mathrm{MPa}$ at 35 to $75 \mu \mathrm{m}$ below the surface. A slight different stress distribution is observed for the cutting direction (CD). While the surface tensile layer, $20 \mu \mathrm{m}$, is thicker, the surface tensile stress, $265 \mathrm{MPa}$, and the subsurface compressive peak, $313 \mathrm{MPa}$, are somewhat lower than those of the cutting direction. The existence of the out of plane shear stress parallel to FD causes a split in the d-sin $2 \psi$ distribution, which results in a larger experimental uncertainty for CD measurement up to $100 \mu \mathrm{m}$ below the surface. On the other hand, the depth under stress is similar for both FD and CD and corresponds to the penetration depth of plastic deformation indicated by the FWHM curve. Such a characteristic residual stress distribution can be attributed to the surface plasticity causing a deep compressive residual stress zone in the surface, modified by a tensile residual stress field in a thin surface layer [9].
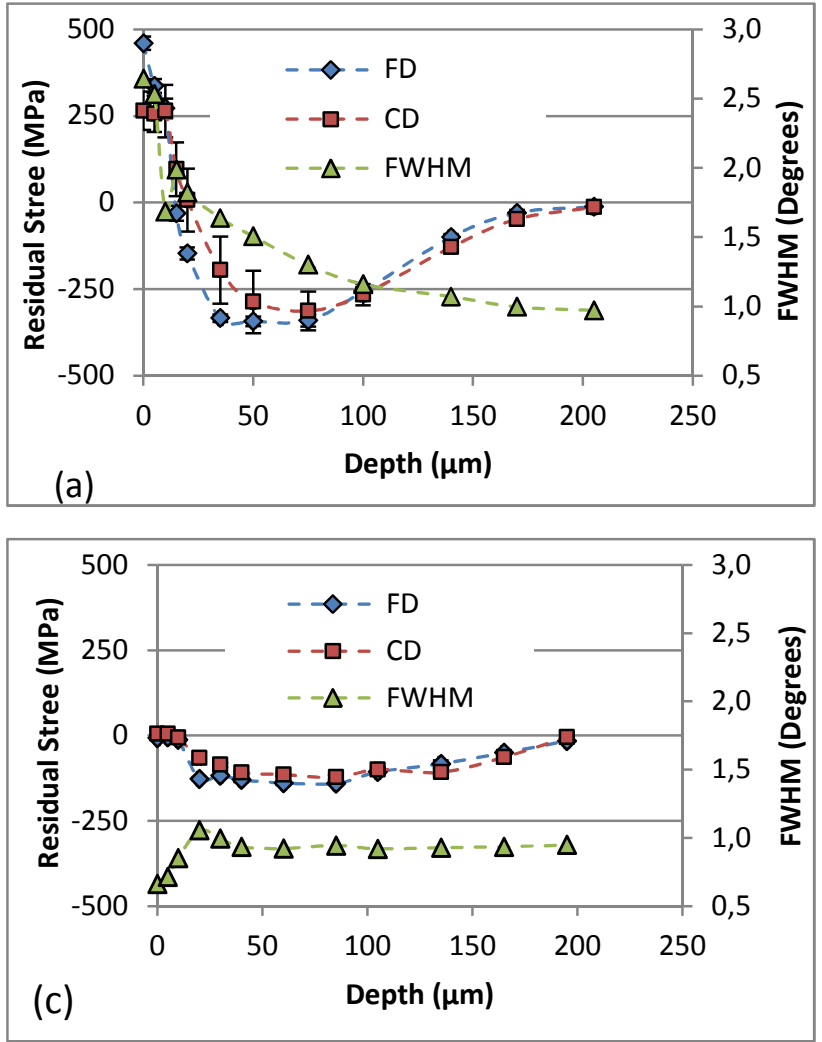

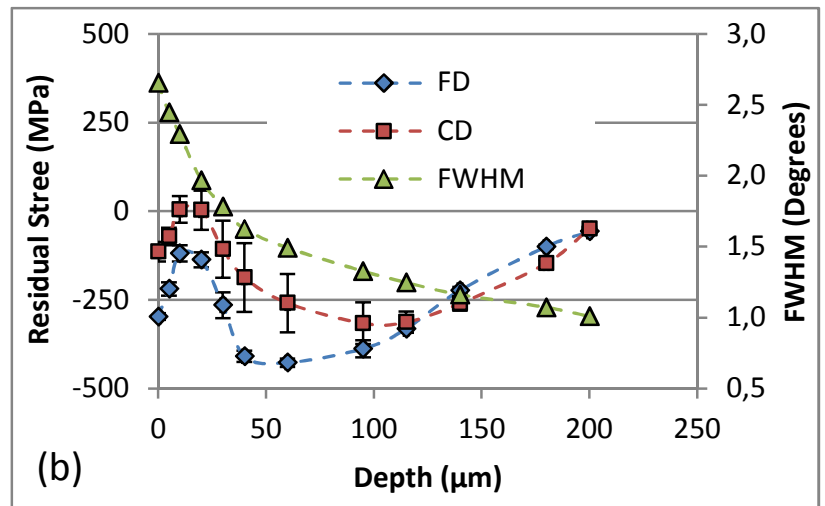

Figure 2 Depth distribution of residual stress and peak width in (a) as machined, (b) vibration treated and $(\mathrm{c})$ heat treated sample.

\subsection{Microstructural Changes}

Fig. 3 compares the microstructure of the surface layer for the different conditions. Unindexed poor Kikuchi patterns (zero solutions) related to either grain boundaries or severe plastic deformation are shown as grey pixels in the colored EBSD maps (Figs.3(f)-(h)) but black pixels in the greyed EBSD maps (Figs. 3(i)-(k)). Because of strong strain contrast of the tempered martensite, transition to the unaffected bulk can't be clearly defined by ECC. Nevertheless, Fig. 3(a) reveals significant microstructural alternation, in the form of grain refinement and distortion, in the subsurface of the machined ring. The very fine grained structure (Figs. 3(a), (c) and (f)) with a high percentage of zero solutions is observed in a depth up to $5 \mu \mathrm{m}$. The formation of such a layer can be ascribed to severe plastic deformation and possibly local heating during turning [9]. 


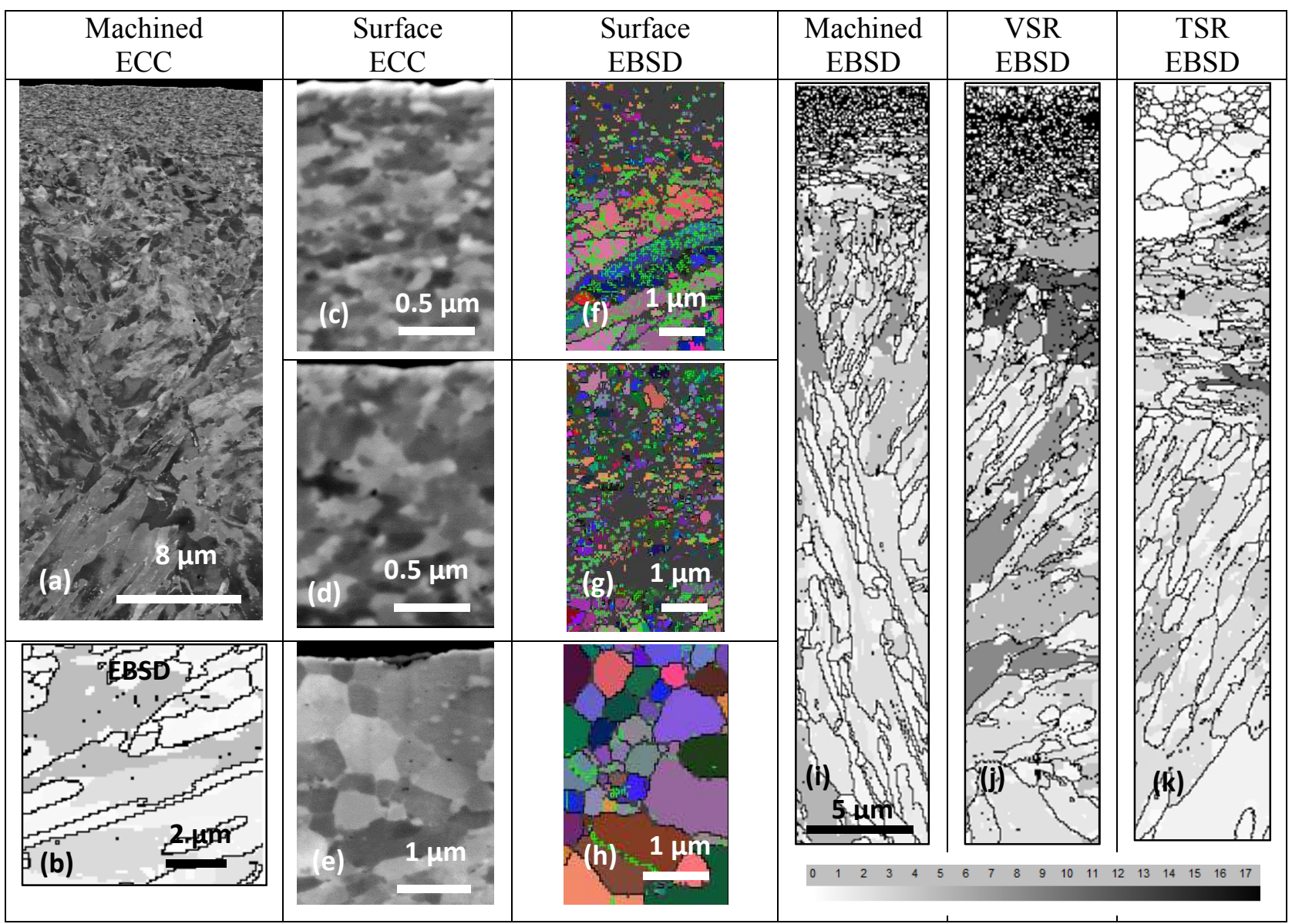

Figure 3 ECC images for the machining affected depth (a) and the outer surface layer of the machined (c), VSR (d) and TSR (e) with corresponding EBSD maps in (f)-(h) (zero solutions in grey). EBSD maps in (i)-(k) compare GAM in a surface layer of about $30 \mu \mathrm{m}$ and (b) is from the unaffected bulk, with zero solutions in black. The cutting surface is always on the top. Grain boundaries (black lines) in the EBSD maps are defined as a misorientation over $7.5^{\circ}$.

Microstructure gradient in the subsurface of the VSR ring is similar to that in the machined ring. A close comparison of the EBSD maps (Fig.3(f) and (g)) seems to indicate somewhat increased plastic deformation in the near surface layer; the fine grained surface layer shows a slightly larger thickness and a higher concentration of zero solutions. Furthermore, Fig. 3(j) also reveals higher grain average misorientations (GAM) in the subsurface below the fine grained layer than Fig. 2(i). GAM is calculated by averaging all misorientations measured between two neighboring pixels in the grain. For a given material increased plastic deformation can lead to increased GAM [10]. For comparison, GAM distribution in the unaffected bulk of the as machined ring is given in Fig. 3(b).

Significant microstructural changes of the machining affected depth are observed after the TSR treatment (Figs. 3(e), (h) and (k)). Essentially full recrystallization occurs in the previously fine grained layer and partial recrystallization in the depth below. No noticeable changes are however induced in the bulk unaffected by the machining. Hardness measurements that are not reported here gave similar results for all the three rings.

\subsection{Discussion}

It has been shown that VSR effectively reduced surface residual stresses and also decreased somewhat subsurface residual stresses due to inhomogeneous plastic deformation such as in cold rolled [5] or bent [4] bars. Similar influence was also reported on residual stresses of thermal origin, for example in welds [3]. Different from these studies, the application of VSR on the machined rings reverses the surface machining residual stresses from tension to compression and at the same time increases somewhat the subsurface compressive stresses along FD. On the cold rolled bar [5], Walker et al. reversed the residual stress field from cold rolling, turning the surface layer into low 
compression and subsurface into tension by applying vibratory bending that caused global yielding of the bar. Global yielding from bending load is not the case here as both the FWHM in Section 3.1 and microstructure observation in Section 3.2 did not reveal massive plastic deformation in the vibrated ring. Besides, similar changes are also observed in both $C D$ and FD, but bending of the ring in its width is difficult. The microstructural examination in Section 3.2 indicates that local/microscopic plasticity is still responsible for the relaxation of the surface tensile residual stresses. It is postulated that by inducing a local, initial yielding and then creep in the vibration cycles followed [6] the applied amplitude stress together with the high local tensile stresses removes the inelastic tensile strains of thermal origin in a thin surface layer. This results in the restoration of part of the surface compressive residual stresses from the machining induced surface plasticity.

It is difficult to explain changes in the subsurface compressive residual stresses. With a lower degree of strain hardening, yielding and creep under compression could have occurred especially at the depth where peak compressive stresses exist and reduced their magnitude. It is possible that local plastic deformation in subsurface is more difficult than in the very surface layer. It is also possible that due to a lower temperature, fine carbides of the tempered martensite are not affected by the cutting heat and prevent massive dislocation movement during the VSR treatment. Further investigation by experiment and modelling are needed to fully understand the influence of VSR on machined components which do not only have complex residual stresses of thermal and mechanical origins but also have a sharp gradient of microstructure and strength.

Relaxation of machining induced residual stresses by TSR annealing also involves local plasticity. At the annealing temperature, the strength of the material drops to a much lower value. Residual stresses in the surface and subsurface layers are reduced through rearrangement of dislocations, a process which also removes most of the plastic deformation from machining. Because of the large elastic energy from machining, recrystallization occurred in the severely deformed thin surface layer, as shown in Fig. 3(e) and (h) and results in complete relaxation of the surface tensile residual stresses.

From the point of view of fatigue resistance, VSR, which hardly affects the hardened microstructure in the surface layer and converts the surface tensile residual stress to compressive, may be more favorable than TSR. However, it should be borne in mind that VSR may induce cyclic damage especially under excessive treatment time or amplitude, which can be detrimental for the fatigue life.

\section{Conclusions}

Tensile residual stresses in a thin surface layer and compressive residual stresses in a thicker subsurface layer were observed in the as machined ring. Severe plastic deformation occurred in the surface layer with a fine grained surface layer.

The vibratory treatment replaced the tensile residual stresses from machining with low compressive residual stresses and increased the subsurface compressive stresses in the cutting direction without inducing significant microstructural changes in the machining affected zone. The reversion of residual stresses in the surface layer is attributed to plasticity induced by the superposition of amplitude stress and the local residual stresses.

The stress relaxation annealing treatment completely removed the surface tensile residual stresses and substantially reduced the subsurface compressive residual stresses. Microstructural alterations occurred in the machining affected layer, with essentially full recrystallization in an outer layer which led to the full relaxation of the tensile residual stresses.

Both the residual stress analysis and microstructural observation seem to indicate that VSR may be more favorable than TSR regarding fatigue resistance of components.

\section{Acknowledgement}

Ms Amaia Bretos Cortazar and Mr Daniel Lopez Gomez are acknowledged for the hardness measurements. Faculty Grant AFM (Faculty Grant SFO-MAT-LiU\#2009-00971) at Linköping University is also acknowledged. 


\section{References}

[1] O. Voehringer, RELAXATION OF RESIDUAL STRESSES BY ANNEALING OR MECHANICAL TREATMENT. Adv in Sur Treat, Technol - Appl - Eff. 4 (1987) 367-396.

[2] R.A. Claxton, Vibratory stress relieving - an effective alternative to thermal treatment for component stabilisation, Heat Treat. Met. 18 (1991) 53-59.

[3] M.C. Sun, Y.H. Sun, R.K. Wang, Vibratory stress relieving of welded sheet steels of low alloy high strength steel, Mater Lett. 58 (2004) 1396-9.

[4] R. Dawson, D.G. Moffat, Vibratory stress relief: a fundamental study of its effectiveness, Transactions of the ASME. Journal of Engineering Materials and Technology. 102 (1980) 169-76.

[5] C.A. Walker, A.J. Waddell, D.J. Johnston, Vibratory stress relief - an investigation of the underlying processes, Proc. Inst. Mech. Eng. Part E J. Process Mech. Eng. 209 (1995) 51-58.

[6] S. Kwofie, Plasticity model for simulation, description and evaluation of vibratory stress relief, Materials Science \& Engineering: A (Structural Materials: Properties, Microstructure and Processing). 516 (2009) 154-61.

[7] X. Dong, X. Zhao, S. Yu, X. Zhou, D. Ren, Y. Zhai, Comparative study on vibratory stress relief (VSR) and thermal stress relief (TSR) of cylinder wallboard, Adv. Mater. Res. 331 (2011) 460-464.

[8] I.C. Noyan, J.B. Cohen, Residual Stress Measurement by Diffraction and Interpretation, (1987).

[9] Lin Peng, R., Zhou, J.M., Johansson, S., Billenius, A., Bushlya, V, Stahl, J.-E., Influence of Dry Cut and Tool Wear on Residual Stresses in High Speed Machining of Nickel-Based Superalloy, To Appear in Materials Science Forum. (2013).

[10] S.I. Wright, M.M. Nowell, D.P. Field, A Review of Strain Analysis Using Electron Backscatter Diffraction, Microscopy and Microanalysis. 17 (2011) 316-29. 